\title{
Educación, tecnología y nuevos espacios para el trabajo colaborativo, para el apoyo a la docencia
}

\author{
doi: 10.33264/rpa.202001-07 \\ Karen Gutiérrez Dumont \\ Escuela de Arquitectura \\ Facultad de Arquitectura, Diseño y Artes Visuales UNIACC
}

\section{Resumen}

Lograr comprender la relación entre educación y tecnología como una experiencia docente facilitadora de procesos de aprendizaje y adquisición de conocimientos reflexivos en torno al hacer académico en educación superior, es difícil de resolver, sería trascendental definir conceptos previos que colaboran en la definición de la conformación de un proceso de aprendizaje, que son acciones y estados necesarios para que un individuo logre obtener conocimientos. Lo importante de esto es preguntarse, ¿la tecnología es un aporte a la educación?, ¿cuál sería su aporte?, ¿cómo la transformación digital, la industria 4.0 han cambiado la metodología y procesos de conocimiento en la educación? Este camino que lleva tiempo en construcción, tendrá mucho por hacer y nos permitirá avanzar respecto a nuevas metodologías y formas de mejorar los procesos de aprendizajes.

Palabras claves: Tecnologías, educación, trabajo colaborativo, transformación digital, industria 4.0., virtualidad, entornos digitales.

\begin{abstract}
Achieve to comprehend the relationship between education and technology like a facilitator of learning processes for teacher experience and acquire reflective knowledge around academic superior education is very difficult to resolve, it is transcendental to define previous concepts that collaborate with the meaning of conformation of a learning process, there are actions and necessary states for an individual to obtain acquaintance. The importance of asking oneself: Is technology a contribution to education? What is its contribution? How does the digital transformation, the 4.0 industry change the methodology and processes of knowledge in education? This path, that has taken time to be built, has much to do and will permit us to move along regarding new methodologies and ways to making learning processes better.
\end{abstract}

Keywords: Technologies, education, collaborative work, digital transformation, industry 4.0., Virtuality, digital environments. 


\section{Introducción}

Hoy vivimos en un mundo donde la comunicación ha cambiado, nos movemos mucho más en entornos digitales y van decreciendo los entornos de interacción directa entre las personas. Habría que preguntarse por qué cada vez más se prefieren los medios digitales para los momentos de interacción.

Se escucha a menudo decir que la tecnología acerca virtualmente, que permite ir donde no es posible ir, conocer lo que no se alcanza a tocar, y de alguna manera, tiene la facultad de poder acercar a lugares del mundo sin estar físicamente en él. Además permite y da facilidades en cuanto a la comunicación brindando una alta variedad de plataformas virtuales de comuniación. De alguna manera, es posible hoy encontrar un sinfín de herramientas tecnológicas que van a permitir facilitar cierta problemática y hacer, dependiendo del área en que se esté revisando y/o la tecnología de la que se esté hablando.

Un ejemplo actual, es lo que se vive hoy, una de las crisis sanitarias mundiales más devastadora del siglo, el COVID-19, más conocido como Coronavirus, que ya ha llegado a 164 países. El Ilamado desde la OMS²3 (Organización Mundial de la Salud) es a quedarse en casa y hacer cuarentena para evitar contagios. El número de contagios a la quincena de marzo del 2020 es de 215.000 , de los cuales 8.000 pacientes han llegado a la muerte ${ }^{24}$ a solo dos meses de su brote en China. Ante esta situación Chile a determinado Estado de Excepción Constitucional de Catástrofe, evitando de este modo que las personas deban salir de sus casas, proponiendo a las empresas el teletrabajo y a nivel educacional implementando mecanismos de educación online como una forma de seguir una normalidad dentro de la catástrofe. En este panorama mundial, el uso de herramientas tecnológicas ha sido fundamental, y son estos medios los que han permitiendo seguir con las actividades habituales, desde el trabajo a distancias, videoconferencias, hasta visitar un museo virtualmente que está ubicado en Europa.

Las tecnologías han sido el motor al cambio actual respecto a muchos procesos y labores que se hacen de manera análoga, solucionando y facilitando el desarrollo del trabajo, y permitiendo que estos se hagan de manera más eficaz. Tener mecanismos de comunicación que faciliten el estar presentes virtual y remotamente, es lo necesario para poder abordar sistemas de trabajo o estudio que dejen acercar y escuchar sin estar físicamente.

\footnotetext{
23 https://www.who.int/es/emergencies/diseases/novel-coronavirus-2019

24 Información extraída de https://www.emol.com/noticias/Internacional/2020/01/29/974946/Mapa-GraficosCasos-Coronavirus-China.html 
Desde la invención de Internet, y en la primera descripción más concreta que se tiene a través de la red realizada en agosto de 1962, queda demostrada su interacción social que podía posibilitar. Esto queda en concreto documentado en los memorandos escritos por J.C.R. Licklider, del MIT (Instituto de Tecnología de Massachusetts). Dichos documentos relatan el concepto de "Red galáctica"25, lo cual queda en evidencia en público en octubre de 1972, con una gran demostración de ARPANET $^{26}$, en la International Computer Communication Conference (ICCC). Esta fue la primera vez que se utilizó esta nueva tecnología de redes demostrada en público, y se introduce el concepto de la aplicación "hot" inicial y el correo electrónico. A partir de esta referencia, se hace necesario comprender puntos de vistas de experto en tecnologías. Fran García, Ingeniero de Soluciones de Google por Education, en el Encuentro Internacional Virtual Educa Puerto Rico sobre “Investigación, Desarrollo e Innovación en Educación del XVII", reflexiona en torno a las dudas y soluciones que la tecnología ha desarrollado y cuáles aplicaciones podrían aportar a la educación. Menciona al respecto, la importancia de desarrollar habilidades y competencias en cuanto a entornos digitales y a tomar importancia en preguntarse, ¿Es real que la tecnología mejora la educación? ¿Mejora habilidades? ¿cuáles son esas habilidades? y ¿cómo mejorarlas mediante la tecnología? ${ }^{27}$ Desde esta mirada, es posible decir que lo más importante es entender y comprender que la tecnología no es el fin, es simplemente el medio, el criterio es humano y jamás podrá ser reemplazado.

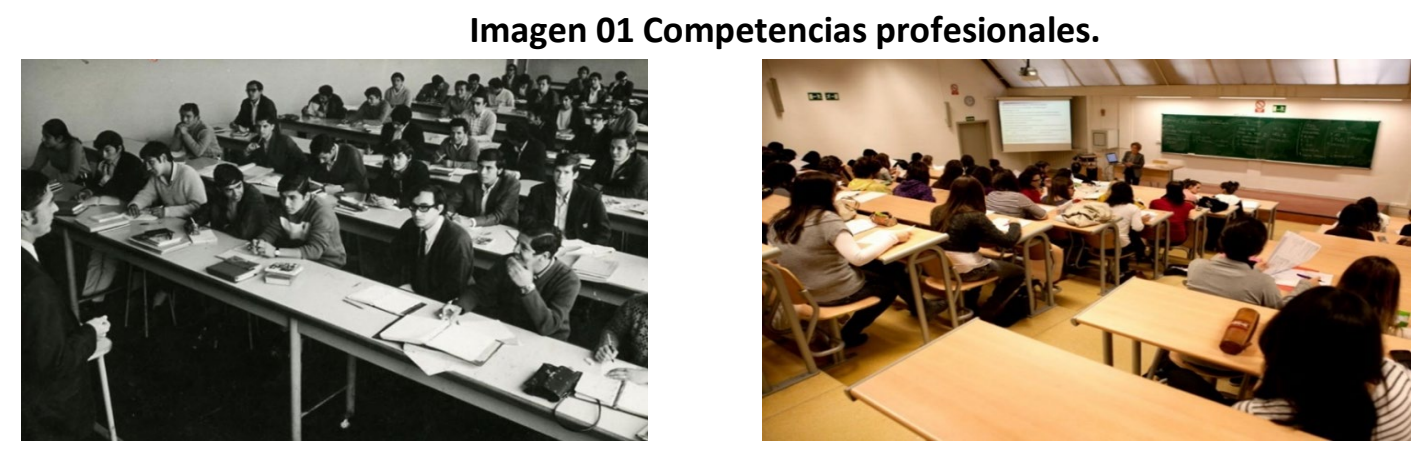

Fuente: Karen Gutiérrez (2020).

La pregunta es, ¿están preparados los alumnos universitarios para la educación del

\footnotetext{
25 Basado en lo mencionado por J.C.R. Licklider quien fue el primero en promulgar en 1962 el concepto de "red galáctica", una red capaz de comunicar usuarios en distintas computadoras. Barataria. Revista CastellanoManchega de Ciencias Sociales.

26 Arpanet (Advanced Research Projects Agency Network) Es una de las redes creadas por encargo del Departamento de Defensa de los Estados Unidos para poder establecer un importante nexo de comunicación entre los distintos Organismos Gubernamentales de la nación. BARATARIA. Rubio Lopez. Tecnologías de intenet (de Arpanet a la 3G). Revista Castellano-Manchega de Ciencias Sociales.

27 Basado en la reflexión expuesta en video Fran García (2016), Ingeniero de Soluciones de Google por Education de https://www.mesaticfid.cl/la-tecnologia-mejora-la-educacion-fran-garcia-virtual-educa-puerto-rico-2016/
} 
futuro? Si bien el primer acercamiento a las tecnologías que permiten acortar distancias y facilitan el trabajo diario fueron comenzadas a ser frecuentadas en el 1972, hoy en el 2020 aún no se observaban grandes cambios entorno a la educación hasta la llegada de la pandemia que apalancó adelantar lo que muchos teóricos de la educación ya comentaban, Jamil Samil fue uno de ellos ${ }^{28}$. Al observar las dos imágenes anteriores, se logran identificar diferencias entre ambas. La primera es del 1918, mientras la segunda es de 2018, la única diferencia que se puede observar es que una está en blanco y negro y la segunda en color, no hay mayores contrastes, de alguna manera se puede concluir que dentro de las aulas de clases en 100 años trascurridos los métodos de enseñanza educacional superior no han cambiado ${ }^{29}$.

Si bien ya es posible entender cuál podría ser la búsqueda en torno a la tecnologías, es importante definir qué busca la educación superior hoy. Hay que preguntarse entonces, qué es lo que esperan las empresas en la actualidad de sus futuros profesionales. La UCA, Universidad Católica de San Antonio de Murcia, explica que dentro de las 10 competencias más valoradas por las empresas las 03 primeras son el trabajo en equipo, la comunicación y la solución de problemas ${ }^{30}$. El mundo es cada vez más global y las capacidades de trabajar en equipo es una de las más importantes, por lo cual es necesario hacer una revisión del contexto en que estamos inmersos para formular estrategias metodológicas que pudieran aportar a los sistemas educacionales actuales para formar los profesionales que las empresas esperan ${ }^{31}$.

Cuando se habla de tecnologías se nos viene a la cabeza, varios conceptos que apoyan y validan a ésta como un puente para facilitar métodos y procesos que hasta hoy se hacían de manera tradicional. Dentro de las tecnologías el concepto de colaboración es uno de los conceptos que es capaz de ser desarrollado de manera eficaz mediante la tecnología.

Es importante pensar en cambios en los paradigmas que determinan la educación, desarrollar un método de trabajo que puedan comenzar con planes de acción,

\footnotetext{
28 Jamil Salmi ya en el año 2013 habla de cómo será la educación superior en siglo en el XXI, el habla de los nuevos desafíos en La Conferencia Magistral impartida por el Mtro. con el tema "Nuevos desafíos para la Educación Superior en el Siglo XXI", es decir los tiempos que vivimos hoy nos están presentando problemáticas que es necesario solucionar. Salmi menciona de como la tecnología tiene una gran implicancia en la solución de problemas como la distancia física, la inclusión, brechas generacionales y económicas, de las cuales las herramientas tecnológicas, nos permiten abordar y solucionarlas, para que la educación logre su objetivo social.

29 Basado en la reflexión expuesta en video Fran García (2016), Ingeniero de Soluciones de Google por Education de https://www.mesaticfid.cl/la-tecnologia-mejora-la-educacion-fran-garcia-virtual-educa-puerto-rico-2016/

30 Basado en artículo publicado por UCAM, marzo 2017. https://online.ucam.edu/

31 Reflexión basada en artículo Tecnología, empleo y el futuro del trabajo, Mayo 2016. Estudio McKinsey Cia. https://www.mckinsey.com/featured-insights/employment-and-growth/technology-jobs-and-the-future-ofwork\#
} 
desarrollar métodos de cómo implementar, definir métodos de evaluación para obtener resultados y proponer sistemas que determinen una mejora continua.

\section{Metodología}

Este documento se ha desarrollado bajo una metodología de investigación fundada en la obtención de información a partir de práctica docente realizada en la carrera de Arquitectura de Uniacc.

Para la obtención de información, análisis y reflexión en torno al tema de central del documento, se realizó primero, bajo la observación y reconocimiento de los actuales sistemas de educación, y como la Facultad de Arquitectura ha podido sobrellevar este tiempo de distanciamiento social bajo metodologías de enseñanza remota y virtual de manera exitosa. Por otro lado, y como una manera de validar las reflexiones anteriormente expuestas, se hace un estudio basado en la teoría y revisión de contenidos, para obtener un marco de referencia fundado en las bases y fundamentación para implementar las tecnologías como parte del hacer docente e incorporarlo a la metodología de enseñanza.

\section{Acercamiento a las tecnologías}

Con la llegada de las Redes sociales como Messenger, Facebook, Instagram, Twiter, Youtube, Linkedln y ahora TicToc, los medios de comunicación han logrado ser mucho más inmediatos y de rápida difusión, es decir, no solo estos medios propician la comunicación, sino la difunden de manera más rápida dado el flujo de rapidez de información que es posible generar y transportar mediante internet.

En cuanto a educación superior es posible preguntarse cómo enfrentar estos cambios respecto a las experiencias académicas y cómo el hacer docente permite evidenciar y entender el hacer de la docencia desde un contexto real.

Desde esta mirada, es importante considerar situaciones de las cuales es posible reflexionar en torno a la actividad docente. En este sentido hay dos grandes temas que son interesantes de revisar, y dar respuesta a cómo se debe transformar la educación en cuanto a medios tecnológicos actuales y sus metodologías correspondientes, esto referido al cambio cultural al que nos vemos enfrentados respecto a las tecnologías, los nuevos medios de comunicación versus la educación tradicional, y otro gran propósito que nace a partir de éste es resolver un óptimo proceso de aprendizaje respecto a estos nuevos medios de comunicación, permitiéndo enfrentarse a este nuevo alumno que viene con otros métodos de aprendizajes previos y que ha adoptado nuevas formas de aprender empíricamente.

Entonces es posible especular que la práctica docente debe basarse en un hacer contextualizado a partir de la reflexión propia de la experiencia académica, la cual 
tiene como objetivo ser un facilitador de conocimientos. Desde esta mirada, ser conscientes de los aprendizajes propios de la docencia, para posteriormente traspasarlos, permite entender la construcción del conocimiento curricular y la didáctica de los alumnos en un contexto real y contemporáneo.

\section{Objetivo general}

Ante lo anteriormente mencionado, es importante entonces determinar y entender objetivos generales que permitan dar líneas de entendimiento entorno a la educación superior y las tecnologías. Este será:

- Reflexionar en torno a las tecnologías y la educación como una experiencia docente facilitadora de procesos de aprendizaje y adquisición de conocimientos reflexivos en cuanto al hacer académico en educación superior para formular posibles propuestas para abordar el tema en el contexto actual.

Para ello es necesario comprender objetivos específicos como:

- Mencionar conceptos que elaboran y conforman un proceso de aprendizaje necesario para que un individuo logre obtener conocimientos.

- Reflexionar entorno a si la tecnología es un aporte a la educación.

- Comprender que la transformación digital y la industria 4.0 han cambiado nuestro contexto habitual, y con ello cambiará las metodología y procesos de conocimiento en la educación.

Para poder llevar a cabo el desarrollo de estos objetivos es necesario incluir una serie de conceptos a revisar tanto desde la docencia, la organización educacional, como nuevos conceptos que hoy nos incorporan las nuevas tecnologías.

\section{Modelo educativo, proyecto y contexto político y actualizado, narrativas reflexivas respecto del aprendizaje}

Para comenzar a comprender cómo este cambio es un camino a la trasnformación y afecta el entorno académico, es necesario dar revisión a conceptos que determinan este hacer. Uno de estos conceptos es el Modelo educativo. Al reflexionar respecto a las implicancias que definen el Modelo educativo, podemos decir que es un método para orientar y definir la pedagogía en un entorno de enseñanza, concretando los principios que se fundan en el Proyecto educativo, el cual hace referencia al potencial político conceptual que se desarrolla a través de este modelo educativo para cumplir sus propósitos y objetivos ${ }^{32}$.

\footnotetext{
32 Basado en lo mencionado en PDF, Modelo Educativo UNIACC. Vicerrectoría Académica UNIACC. (2016). 
El Proyecto educativo, a su vez, debiera siempre ser abordado desde una mirada humanista, respecto a la concepción de sujeto, en donde es la persona lo más importante, son los sujetos que están relacionados al contexto, a lo multidimensional y al conocimiento ${ }^{33}$ que se funda en ello, prestando mayor importancia a la reflexión en torno al sujeto antes que cualquier otro tipo de aprendizaje dado.

Es posible revisar estas reflexiones en torno al sujeto desde lo mencionado por Durkheim (1976), en Educación como sociabilización, entiendo la importancia de que la educación sea vista como un hecho social que implica un reconocimiento de la sociedad y del individuo ${ }^{34}$.

Desde esta reflexión, es posible comprender qué importante es definir y comprender el sujeto como aquel que se relaciona con el medio y que es capaz de lograr un conocimiento, dando pie al saber y de este modo llegar al objetivo planteado por el Modelo educativo, el cual plantea que es necesario que el sujeto sea reconocido como tal, entendiendo que es capaz de generar aportes a la sociedad, al contexto, con una mirada crítica, creativa, contribuyendo a formar sujetos con posibilidad de lograr aprendizajes significativos 35 .

\section{La necesidad de comprender los métodos más esenciales en el proceso de aprendizaje, el aprender a aprender.}

Comprender la tecnología en relación a la educación superior, permite preguntarse si los alumnos están preparados para entender estos nuevos posibles mecanismos de enseñanza. De este modo es necesario hacer revisión para vislumbrar la interacción multidimensional, a la creación, el descubrir y la experimentación, en donde es posible dar pie al aprender a aprender y comprender los procesos que contribuyen asimilar esta transformación. Para ello es necesario abrir los horizontes a lo que pasa en la realidad desde la mirada global e interdisciplinaria, permitiéndose promover un ambiente colaborativo e integral para los alumnos, dejando espacio para la reflexión de ellos desde sus aportes personales, abriéndose al campo de la investigación e experimentación personal, convirtiendo esta experiencia, en una transformación facilitadora de conocimientos para que sea una guía de articulación de éstos. Es así como el Modelo educativo debería comenzar a transformarse, enmarcarse y promover aprendizajes desde una mirada renovada, creativa e innovadora, y expandirse a las clases, a los alumnos y reconocer el contexto sociocultural donde están insertos, incentivando un objetivo transformador en beneficio de todos,

\footnotetext{
33 Basado en lo mencionado en PDF, Modelo Educativo UNIACC. (Vicerectoría Académica UNIACC, 2016).

34 Basado en Capítulo 4 “La educación: su naturaleza, su función”., Emile Durkheim. (1976). En Educación como socialización.

35 Basado en lo mencionado en PDF, Modelo Educativo UNIACC. Vicerrectoría Académica UNIACC. (2016). 
manteniendo el compromiso por él y con él ${ }^{36}$.

Este proceso donde se realiza el reconocimiento a dicha transformación de movimiento y creación de conocimiento, es el ciclo de aprendizaje, el cual también debiera ser entendido desde el reconocimiento de los elementos psicológicos y socioculturales que lo componen, y de este modo desarrollar experiencias en los estudiantes, donde ellos sean el centro del aprendizaje, y que de alguna manera, éstos deban siempre relacionarse con el entorno, permitiéndoles construir aprendizajes con su propia voluntad. Mediante el aprendizaje es posible reconocer procesos como el reconocimiento de aprendizajes previos, la problematización, la práctica reflexiva, la resignificación, y por último la creación-transformación, ahora el estudiante tiene la capacidad de transformar y contribuir al desarrollo de su entorno sociocultural, con una mirada crítica y reflexiva37.

Desarrollar y potenciar el Aprender a aprender, como un concepto que promueve el aprendizaje, y hacer que cada individuo pueda ser consciente, primero desde su individualización a un proceso de aprendizaje, luego a desarrollar y/o adquirir técnicas, destrezas, estrategias y habilidades que permitan tanto a alumnos como a profesores, comprender desde ellos mismo, reconociendo y aprendiendo a pensar en cuanto al análisis de la realidad actual, enfrentandose a los desafíos con criterio y poder de decisión, a nuevos métodos de enseñanza, potenciando el carácter crítico de los estudiantes y desarrollando procesos de aprendizajes íntegros y transversales.

Desde esta mirada se torna fundamental preguntarse por la importancia de evolucionar en cuanto a las metodologías de aprendizajes, por medios tecnológicos que faciliten los medios de comunicación entre los alumnos y el profesor, y además asumir la tecnología e innovación en cuanto a la educación, como una herramienta que permita crear, y/o abrir a nuevos campos de acción.

Aprovechar los medios comunicacionales digitales, tecnologías e innovación induce a la investigación y permite descubrir e investigar, labor que muchas veces ha sido olvidada por los docentes y por las instituciones educacionales.

\section{Los pilares del conocimiento empírico en la actualidad}

El proceso de potenciar y comprender el aprender a aprender, implica el uso adecuado de estrategias metacognitivas, las cuales deben basarse en potenciar el propio pensamiento y reconocer procesos del analizar, pensar y aprender. El

\footnotetext{
${ }^{36}$ Reflexión basada en lo mencionado en PDF, Modelo Educativo UNIACC. Vicerrectoría Académica UNIACC. (2016).

37 Basado en lo mencionado en PDF, Ciclo de Aprendizaje UNIACC. Vicerrectoría Académica UNIACC. (2017). 
desarrollo de este proceso es necesario para promover el aprendizaje, el aprender a aprender y lograr que utilicen adecuadamente las estrategias metacognitivas. De alguna manera, potenciar la capacidad de crear y transferir, reales estrategias facilitadoras en cuanto a la solución de problemas, mediante la concreción y desarrollo de las capacidades intelectuales, les permitirá a los alumnos, poder enfrentarse a problemáticas de toda índole y resolverlas desde un plano más reflexivo, lo cual les brinda un grado de autonomía en cuanto a sus propias creencias y criterios, quedando supeditados a sus conocimientos previos, a la incorporación de nuevos conocimientos y las conclusiones respecto a la reflexión de ambos. Este estado superior de análisis, permite desarrollar la mirada crítica del tema en cuestión y sobrepasa el proceso de adquisición del conocimiento propiamente tal, dejándolo atrás, avanzando a un plano reflexivo superior, que va más alla de solo tener que adquirir conocimiento, abriendo paso a observar, pensar y reflexionar.

\section{Educación y tecnología}

Incorporar en la docencia entornos basados en la tecnología y pensar en ella en pro de la educación, hace años algo innimaginado en el proceso educativo. La relación entre educador y educando, se funda en la capacidad que se tiene de comunicar entre ambos, sin embargo y en el contexto actual, ha sido necesario comprender y adaptar los cambios tecnológicos que hoy suceden e incorporarlos a la práctica docente.

En cuanto a las tecnologías, éstas hoy han contribuido e incrementado la comunicación social de masas ${ }^{38}$, transformándola en un nuevo entorno digital, con plataformas abiertas de comunicación, permitiendo que ésta fluya de manera circular y no vertical como en la antigüedad. De alguna manera las tecnologías han propuestos que la información hoy las podemos manejar libremente mediante internet dejando atrás las jerarquías y sistemas piramidales en cuanto al manejo de ésta. Los medios de comunicación y la información han hecho que nuestra forma de acceder al saber y de conocer haya cambiado, lo que ha implicado un cambio en los procesos de aprendizaje, además de un cambio en la manera de mostrar este aprendizaje, y por supuesto al docente permitirle ampliar e implementar nuevas maneras de relacionarse con alumnos y impulsando cambio sustancial en los métodos de enseñanza.

Un ejemplo muy frecuente, se observa en carreras académicas dedicadas a la plástica, donde es formado el profesional desde una mirada más empírica-técnica, potenciando el acercamiento al análisis. Es muy usual que en Escuelas de

\footnotetext{
${ }^{38}$ Basado en La comunicación de masas y las otras comunicaciones. Paradojas y análisis Comunicar. Sánchez Martínez, Mariano. núm. 14, marzo, 2000 Grupo Comunicar Huelva, España. 
arquitectura los primeros años de estudios, se les solicita a los alumnos grandes cantidades de dibujos o croquis para que suelten la mano y aprendan dibujando desde el real, de este modo se les permite no solo que tengan destreza en el hacer, sino que logran poder desarrollar el poder de análisis en torno a un espacio definido y desarrollan la observación. Esta metodología no permite y usualmente está restringido el uso de tecnologías, priorizando el hacer análogo (a mano). Pues bien, hoy, donde 10 de cada 10 alumnos lleva en su bolsillo un celular con cámara, pensar en solicitar que elaboren un croquis de manera in situ y prohibir el uso de tecnología como apoyo, o que no fotografíen para luego reproducir la imagen en casa, que es lo que todo docente haría, podría ser un error. Sería más correcto enseñar las posibilidades que les brinda la fotografía y el uso de herramientas asociadas para poder después entender el croquis, de este modo se podría comprender que el lente de la cámara de un celular sirve para enmarcar, memorizar, agilizar los tiempos en terreno, etc. Esto es comprender que hoy se vive con la tecnología y se debe adaptar nuestras forma de aprendizaje y construir nuevasformas de crear conocimiento, desde lo innovador que las herramientas digitales nos aportan y con nuevos mecanismo de metodologías.

\section{Virtualidad}

Es así como el concepto de virtualidad, en el caso de programas académicos presenciales y semipresenciales, permite generar espacios de comunicación atemporal, donde los docentes, pueden desarrollar contextos nuevos propicios para las relaciones y siendo los profesores, facilitadores de conocimientos, y la educación debe garantizar la comunicación, la interacción, y la entrega de información, como también propiciar procesos de aprendizaje en cuanto nuevos mecanismos y metodologías de educación respecto de estos nuevos medios de comunicación.

La educación y la virtualidad pueden complementarse y beneficiarse una de otra, la educación puede mejorar sus procesos de enseñanza y aprendizaje, y la virtualidad se enriquece por el proceso educativo y de comunicación.

Es cierto que la relación que llevan ambas es compleja, educación y virtualidad, pero para que esto exista, es necesario hacer revisión de la experiencia que tiene cada docente y buscar el modo de adecuar las nuevas tecnologías de la información y de la comunicación permitiendo que siempre estén al servicio del estudiante, es decir, al proceso de aprendizaje y siendo utilizadas como herramientas.

De este modo, comienzan a ser cada vez más familizares conceptos nuevos como entornos virtuales, o más conocidos como foros, que son y permiten tener una comunicación formal en las asignaturas online y semipresenciales, como es en el caso 
de carreras de la Universidad UNIACC donde normalmente son implementados por los profesores. Estos entornos virtuales deben ser entendidos como espacios de comunicación, permitiendo que exista una relación entre estudiantes, profesores y entre todos ellos a la vez. La idea es que sea comprendido como una comunidad, siempre dirigido y articulado desde la práctica docente. Estos entornos virtuales de aprendizaje son el lugar donde se da el estudio del que participan alumnos articulado por los profesores, siendo necesario comprender que desde esta experiencia, este nuevo espacio virtual se presenta abierto y propicio a la creatividad, tomándolo como un reto que aporta con nuevas herramientas para mejorar los procesos educativos y renovar los métodos de aprender, descubrir y potenciar metodologías que estén a la vanguardia de la industria 4.0 y la transformación digital. Queda mucho aun por aprender y potenciar de estas herramientas digitales.

\section{Educación y brecha generacional, tecnologías como campo de acción a través del curriculum.}

La brecha generacional es un tema importante a tratar respecto a cómo los campos de las tecnologías podrían dar cabida a un espacio neutral de construcción de conocimientos aportando con sus herramientas digitales para nivelar las diferencias de conocimientos previos y empíricos que las diferentes generaciones de estudiantes puedan tener.

Para entender la relación es necesario revisar el desarrollo de aspectos a considerar respecto al diseño, análisis y desarrollo del curriculum, entendido como un espacio mediador, junto a las tecnologías y métodos de enseñanza integrados para diferentes generaciones de estudiantes, y cómo la configuración del curriculum debe contener aspectos técnicos, estándares generales y prácticos desde una perspectiva crítica.

La construcción de saberes previos, son habitualmente formados y desarrollados bajo métodos educativos abordados desde la realidad del estudiante, en la época en que fueron adquiridos. Hoy la conformación de saberes definida por el presente, es una etapa donde las tecnologías son las protagonistas, inscribiendo un cambio cultural y social influyendo en el alumno que se forma al día de hoy.

Seguramente, los docentes de hoy que se enfrentan a esta transformación, son el resultado inconsciente a lo que está sucediendo. Cada vez se hace más necesario contextualizar el momento en donde se está inserto y preguntarse metodológicamente cómo se está articulando los saberes y la construcción de conocimientos entregados a los alumnos en esta nueva forma de comunicar y entender la vida, la cual se desprende del uso de las tecnologías como movimiento social cultural. No se puede simplemente obviar que estos cambios repercuten en las formas de aprender, en la diversidad de alumnos, y cómo el docente debe enfrentar esta diversidad. Un ejemplo claro en cuanto a diversidad y brecha generacional es las 
diferencias entre, los alumnos de la llamada generación $X$, aquellas personas nacidas entre 1960 y 1980 aproximadamente ${ }^{39}$, que son aquellos profesionales o técnicos con asuntos pendientes o vienen en búsqueda de llegar cumplir sueños pendientes respectos a sus vidas profesionales ${ }^{40}$, la tecnología es algo adquirido con el tiempo, distintos a la generación millenials o generación Y, nacidos entre 1981 a 1996 aproximadamente ${ }^{41}$, con condiciones y habilidades diferentes, nacieron siendo nativos digitales, lo que influye respectos a sus carga generacional y saberes previos. De este modo es muy probable que los docentes se enfrenten a brechas generacionales en cuanto al uso de la tecnología y no es de extrañar concluir por ejemplo que la generación $\mathrm{Y}$, portan información y capacidades más cercanas a la tecnología, dado que nacieron y crecieron con ella.

Entender esto desde la implementación de las tecnologías como forma metodológica en el proceso de aprendizaje es fundamental, dado que como concepto de trasmisión de saberes, puede o no acortar la brecha generacional entre la diversidad del alumnado. El uso de distintas herramientas tecnológicas en cualquiera de sus variedades permite enfrentar y acortar estas brechas generacionales y verse beneficiadas ambas, es un campo neutral e imparcial de construcción de conocimientos, permitiendo compartir saberes, como lo son los foros, los proyectos de Humanidades Digitales ${ }^{42}$, u otros entornos digitales.

Pues bien, estas nuevas condiciones se deben enfrentar desde las preguntas qué, por qué y cómo se abordan estos cambios, y probablemente la respuesta será, planificar el cómo se articulan, desarrollan y qué objetivos se utilizarán para entregar estos contenidos, de este modo es indudable pensar en el curriculum.

Para comprender mejor este concepto, se referencia a lo expuesto por J. Gimeno Sacristán en su libro "El curriculum: reflexión sobre la práctica”, donde se menciona el "currículum es la suma de exigencias académicas, valorar los saberes específicos que hablan de una cultura elaborada, haciendo de estos conocimientos un instrumento de progreso, dado que ahora, la sociedad exige mejores preparaciones"43. Entonces, el

\footnotetext{
39 Según lo planteado en la investigación “La brecha generacional digital”, de L Mario Valverde, Alcívar Nivela y Jaime Espinosa de la Universidad de Guayaquil.

40 Definición basada en lo planteado en la investigación "La brecha generacional digital”, de L Mario Valverde, Alcívar Nivela y Jaime Espinosa de la Universidad de Guayaquil.

${ }^{41}$ Definición basada en lo planteado en la investigación "La brecha generacional digital”, de L Mario Valverde, Alcívar Nivela y Jaime Espinosa de la Universidad de Guayaquil.

42 Las Hunidades digitales son "Las Humanidades Digitales es un término que engloba este nuevo campo interdisciplinario que busca entender el impacto y la relación de las tecnologías de cómputo en el quehacer de los investigadores en las Humanidades. También se conoce con los términos de Recursos Digitales para las Humanidades, Cómputo para las Humanidades, Cómputo en las Humanidades, Informática Digital y Cultural e Informática para las Humanidades.¿De qué hablamos cuando hablamos de Humanidades Digitales? RIO RIANDE, Gimena del / Instituto de Investigaciones Bibliográficas y Crítica Textual (IIBICRIT). CONICET gdelrio@conicet.gov.ar

43 Basado en lo expuesto por J. Gimeno Sacristán (2007). Las teorías sobre el currículum: elaboraciones parciales para una práctica compleja. En El currículum: una reflexión sobre la práctica. Madrid: Morata..
} 
diseño, análisis y desarrollo del currículum hoy, es importante de analizar y pensar desde una serie de condiciones que se deben revisar para definir posibles actualizaciones en torno a las tecnologías. Para ello es necesario analizar distintas perspectivas teóricas del curriculum como lo mencionado por Mirtha Abraham, en el texto "Análisis de algunas Perspectivas Teóricas del Currículum", donde aclara la coexistencia de distintas maneras de comprensión del éste ${ }^{44}$, para luego poder definir líneas de acción que apalanquen y potencien la relación en cuanto a los procesos de aprendizaje y la tecnología.

Es fundamental proponer cambios, la enseñanza repite programas de materias que se enseñan a distintas edades, Gimeno (2007) menciona que es importante dar contenidos atractivos de enseñanza, romper las barreras del academicismo y entregar mayor variedad de conocimientos ${ }^{45}$.

Proponer que el currículum se defina en base a las experiencia y al interés de los alumnos, contextualiza en el hoy, en lo que en realidad le preocupa al alumnado. En este sentido es posible permitir reconocer este cambio de paradigma social digital y asumir, de parte de los docentes, propias miradas, desde aspectos estándares, y permitiendo a los métodos de enseñanza que evolucionen en torno a ello.

En cuanto a la diversidad de alumnos, tenderá a neutralizar sus diferencias generacionales, proceso que es posible hacerlo desde las alta variedad de herramientas digitales que hoy existen, eso lo hace atractivo y atingente y lo desde espacios y contenidos neutrales de enseñanza, es decir espacios que se enfoquen y tengan una dirección más reflexiva y crítica en pro del diálogo de entender la sociedad en la que vivimos para que sean un real aporte a ésta.

\section{Posibles líneas de acción en la implementación de las tecnologías en la educación superior.}

Además de lo influyente que pudiera ser la reformulación del currículum como apalancador de las tecnologías en la educación, sería importante poder potenciar otras acciones específicas que se focalizan en la implementación de infraestructura y procesos de formación:

- Potenciar y desarrollar la investigación y uso de plataformas virtuales de formación continua de docentes.

- Potenciar las capacidades en los docentes en cuanto al uso de tecnologías como herramientas que faciliten el aprendizaje en alumnos.

\footnotetext{
${ }^{44}$ Basado en lo mencionado por Mirtha Abraham (2000). Análisis de algunas Perspectivas Teóricas del Currículum.

45 Basado en lo expuesto por J. Gimeno Sacristán (2007). Las teorías sobre el currículum: elaboraciones parciales para una práctica compleja. En El currículum: una reflexión sobre la práctica. Madrid: Morata. 
- Desarrollar y potenciar estrategias y metodologías docentes centradas en el alumno respecto a las herramientas tecnológicas con un fin educacional.

- Incorporar a los procesos de aprendizaje de los alumnos, tecnología como una manera de mejoramiento de la gestión pedagógica.

- Promover la colaboración y procesos de participación, enseñanza y aprendizaje interactivo, abordadas desde las tecnologías.

- Desarrollar e incorporar entornos virtuales de comunicación como parte del proceso de aprendizaje. ${ }^{46}$

Gimeno (2007) menciona la importancia de apalancar aspectos intelectuales, físicos, emocionales y sociales para el desarrollo de cada persona ${ }^{47}$. Entonces es necesario abrir y derribar las fronteras de las tecnologías y permitir que entren en el diseño, desarrollo y análisis del currículum. Dewey (1998) lo reafirma mencionando que el método no es el medio para algún fin, sino es parte de un todo mucho más amplio y con mayor contenido, por eso la importancia de considerar las tecnologías como un medio y no como un fin para el desarrollo de una educación contextualizada y de acuerdo a los cambios, necesidades y contingencias de la sociedad actual ${ }^{48}$.

Para que toda esta transformación suceda, es fundamental integrar en las organizaciones educacionales, tecnologías que sean instrumentos que permitan potenciar procesos de aprendizaje, motivar la formación de docentes actualizados, con competencias y habilidades para enfrentar las exigencias y transformación que vive el mundo actual.

\section{Discusiones y conclusiones}

El objetivo del documento permite reflexionar en torno a las tecnologías, cómo éstas pueden llegar a ser interesantes, atractivas, inspiradoras y motivacionales, y a su vez, permiten la interacción, impactando en el aprendizaje de los alumnos, por la importancia de poder aprovechar la convergencia digital. También es fundamental comprender, que este cambio de paradigma implica una transformación transversal a todos los aspectos que influyen en el proceso de aprendizaje del estudiante, se debe hacer a nivel organizacional, docente y del alumno.

Desde esta mirada se desarrolla un documento centrado en los alumnos y docentes, en donde más allá de los contenidos que se pudieran desarrollar en clases, serán, probablemente, las metodologías las que se han transformado. El conocimiento al

\footnotetext{
${ }^{46}$ Acciones basadas en PDF" Las Tic en la educación superior, innovaciones y retos. Revista Iberoamericana de Ciencias sociales y humanísticas. Vol. 6, Núm. 12 Julio - Diciembre 2017

47 Basado en lo expuesto por J. Gimeno Sacristán (2007). Las teorías sobre el currículum: elaboraciones parciales para una práctica compleja. En El currículum: una reflexión sobre la práctica. Madrid: Morata.

${ }^{48}$ Basado en Democracia y educación de John Dewey. (1998). Tercera Edición. Ediciones Morata. Madrid, España.
} 
exterior de las aulas se transmite de manera distinta, entonces el mensaje entendido, y que debe transmitir el docente, es entregar también contenido de acuerdo a este cambio.

Hay aspectos que desde la organización educacional pueden permitir esta transformación. Gimeno (2007) lo menciona: el curriculum debe ser un legado tecnológico, el cual se expone y ha sido apoyado desde las burocracias de la organización, siendo que éste debería ser la expresión de los contenidos de la enseñanza, transformándose en un aparato gestor del sistema social y educativo ${ }^{49}$. Es ahí la importancia de contextualizar el curriculum, abordando la racionalidad, la crítica y la práctica como un espacio de estructura, orden y control de cómo se articula la construcción del conocimiento, con objetivos claros y atractivos para los alumnos, reconociendo como es el estudiante de hoy, de ese modo transformamos el currículum en un trasmisor de conocimiento íntegro y totalizador pero con opiniones personales respecto a la perspectiva individual de cada alumno, unificando brechas generacionales, configurando contenidos atractivos y referentes a los avances tecnológicos que hoy nos acompañan.

El profesional de hoy debe saber reflexionar, como resultado de un proceso de adquisición de conocimientos previos adquiridos durante un proceso de aprendizaje y la tecnología debe ser la herramienta que les permite descubrir los medios para potenciar estas instancias de reflexión.

Los medios digitales tecnológicos tienen varias deudas a resolver aún, un de estas es lograr el acercamiento en las salas de clases, que se acostumbra tener en la relación de comunicación alumno profesor. Es probable que sean problemáticas o debilidades de parte de las tecnologías que hay que solucionar, por el momento queda comprender el metodo de interacción entre los humanos está cambiando según pasa el tiempo, y que con ello los estudiantes y profesores también han cambiado, ante lo cual es probable asumir que los métodos de enseñanza deban sostenerse en torno a lo que el contexto actual nos presenta.

\section{Referencias}

Abraham, M. (2000). Análisis de algunas Perspectivas Teóricas del Currículum.

Alcívar, M. V., Cornejo, A. N., \& Izquierdo, J. G. E. (2018). Docentes y estudiantes, la brecha generacional digital. Espirales Revista Multidisciplinaria de investigación, 1(7).

\footnotetext{
49 J. Gimeno Sacristán (2007). Las teorías sobre el currículum: elaboraciones parciales para una práctica compleja. En El currículum: una reflexión sobre la práctica. Madrid: Morata. 
Dewey, J. (1998). Democracia y educación. Ediciones Morata.

Durkheim, E. (1976). La educación: su naturaleza, su función. En Educación como socialización. Sígueme.

García, F. (2016) Ingeniero de Soluciones de Google por Education en el Encuentro Internacional Virtual Educa Puerto Rico sobre "Investigación, Desarrollo e Innovación en Educación del XVII," en el año 2016, Centro de convenciones. https://www.mesaticfid.cl/la-tecnologia-mejora-la-educacion-fran-garcia-virtualeduca-puerto-rico-2016/

García, M.; Reyes, J. \& Godínez, G. (2017). Las Tic en la educación superior, innovaciones y retos. Revista Iberoamericana de Ciencias sociales y humanísticas. 6. https://dx.doi.org/12.10.23913/ricsh.v6i12.135

Gimeno, J. (2007). Las teorías sobre el currículum: elaboraciones parciales para una práctica compleja. En El currículum: una reflexión sobre la práctica. Morata.

Lopez, G. (2006). Tecnologías de intenet (de Arpanet a la 3G). Revista CastellanoManchega de Ciencias Sociales.5. http://dx.doi.org/10.20932/barataria.voi5.256

Maturana, H. (1995). La realidad: ¿Objetiva o Construida? Fundamentos biológicos de la realidad. Editorial Anthropos.

Rio Riande, G. (2014). ¿ De qué hablamos cuando hablamos de Humanidades Digitales?. In I Jornadas Nacionales de Humanidades Digitales. Asociación Argentina de Humanidades Digitales.

Sánchez, M. (2000). La comunicación de masas y las otras comunicaciones: Paradojas y análisis. Comunicar. 14. https://www.redalyc.org/pdf/158/15801403.pdf

Santos, M. (2003). La Evaluación como aprendizaje: Cuando la flecha impacta la diana. Narcea Ediciones.

Pascual, E. (1998). Racionalidades en la producción curricular y el proyecto curricular. En Pensamiento Educativo. 23. 
Vicerrectoría Académica UNIACC. (2016). Modelo Educativo UNIACC. Modelo Educativo UNIACC. UNIACC, Santiago de Chile.

Vicerrectoría Académica UNIACC. (2017). Ciclo de Aprendizaje. UNIACC, Santiago de Chile.

Karen Gutiérrez D.

Escuela de Arquitectura. Facultad de Arquitectura, Diseño y Artes. 